\title{
Study on the Influence of Probe'S Position on the Flow Field in Hose-Drogue Refueling System
}

\author{
Xiaofei Zhang*, Mengquan Hu, Dong Zhang, Pengyun Tian and Ning Wang \\ Aeronautics Engineering College, Air Force Engineering University, Xi'an, China \\ * jubeiweihao2@sina.com
}

\begin{abstract}
In the docking process of hose-drogue aerial refueling system, the bow wave effect of an approaching receiver aircraft will produce a strong aerodynamic effect on the drogue which intensify the deviation of drogue and increase the difficulty of the docking. In order to reduce the influence of bow wave effect, different locations of probe are used to reduce the adverse effect. This paper presents a receiver aircraft's bow wave effect modeling method based on potential flow theory and considers the major shape characteristics of the receiver aircraft. This method is used in a probedrogue aerial refueling dynamic simulation system, which combined with the hose-drogue dynamic model and tanker wake dynamic model. The motion characteristics of the drogue when the probe in the different position and of the different length are calculated and analyzed.
\end{abstract}

Keywords: hose-drogue; probe-drogue; bow wave effect; potential flow theory.

\section{Introduction}

The aerial refueling is a power multiplier in modern naval and air force. The hose-drogue aerial refueling system is simple and widely used $[1,2]$. But the hose-drogue system of flexible structure which is highly sensitive to external factors such as atmospheric turbulence, tanker wake and the receiver-aircraft's bow wave. In the actual docking process, there is the problem of the hose-drogue swing, which increased the difficulty of the docking and security risks. The bow wave effect of the probe on the receiver-aircraft is also greatly influenced by the position of the probe on the receiveraircraft. The common position of the probe is shown in Fig. 1.

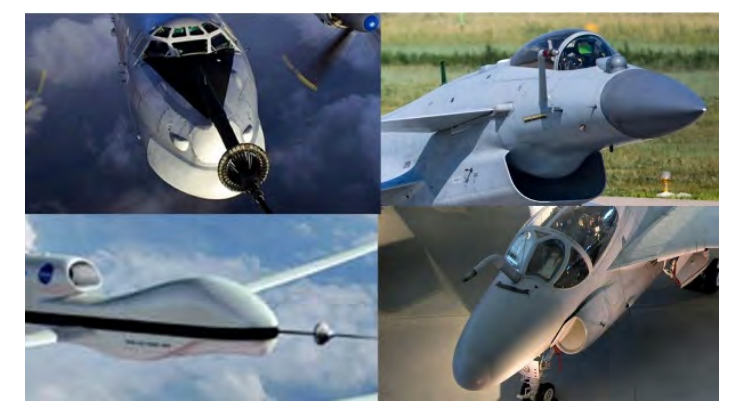

Figure 1. The common position of the probe

In the docking process, when the receiver-aircraft is close to the drogue, the drogue will deviate from the balance position and then quickly return back. This phenomenon is called as the bow wave effect or the forebody effect $[3,4]$. The drogue movement caused by the bow wave brings great uncertainty to the success of the docking. The rise of unmanned aircraft vehicle (UAV) in recent years has opened up new areas for aerial refueling [5]. Therefore, it is required to conduct more in-depth studies on the hose-drogue aerial refueling to ensure the safety of the docking process, whether manned or unmanned.

So far, the research on atmospheric turbulence and tanker wake is relatively mature. However, there is less research on the bow wave effect modeling. Most of people use half Rankine body [6] to simulate the bow wave effect. This method has a small amount of calculation, but also has the problem of accuracy is poor. A CFD simulation was carried out by Wang and Dong [7]. They used the FLUENT software to analyze the variation of bow wave with Mach number and Angle of attack. Khan and Masud [8] used CFD to simulate the aerial refueling of the "Thunder" fighter and analyze the docking strategy of reducing bow wave effect. The CFD simulation results are more reliable. 
However, due to different receiver aircrafts have different complex configuration structure, the CFD simulation requires a large amount of computing resources and time. Moreover, the CFD results are inconvenient for the controller design. NASA Dryden Flight Research Center tested the bow wave effect by F/A-18 receiver-aircraft and estimated the range of the bow wave effect $[5,9]$.

At present, the Study on the influence of probe's position on the flow field in hose-drogue refueling system is inadequate. This paper proposes a fast and efficient receiver aircraft's bow wave effect modeling method based on potential flow theory and considers the major shape characteristics of the receiver aircraft. This method is used in aerial refueling docking simulation system. The motion characteristics of the drogue when the probe in the different position and of the different length are calculated and analyzed.

\section{Frame Definitions and Modeling Assumptions.}

To simplify the modeling of the head wave effect, the following assumptions are made.

(1) In the shape structure of the receiver aircraft, there is a large influence on the flow field near the nose, fuselage, cockpit, main wing and strake wing. Suppose the aerodynamic effects of each structure are independent to each other. According to the superposition principle, the induced velocity of the head wave effect can be expressed as:

$$
\boldsymbol{v}_{\text {bow }}=\boldsymbol{v}_{\text {body }}+\boldsymbol{v}_{\text {cockpit }}+\boldsymbol{v}_{\text {maiwing }}+\boldsymbol{v}_{\text {strakewing }}
$$

Where $\boldsymbol{v}_{\text {bow }}$ denotes the induced velocity produced by the bow wave, $\boldsymbol{v}_{\text {body }}, \boldsymbol{v}_{\text {cockpit }}, \boldsymbol{v}_{\text {mainwing }}$ and $\boldsymbol{v}_{\text {strakewing }}$ denotes the induced velocity produced by the fuselage, cockpit, main wing and strake wing.

(2) Considering the angle of attack of the receiver aircraft is small. So, it can be assumed that the fuselage is axisymmetric.

\section{Bow Wave Effect Modeling}

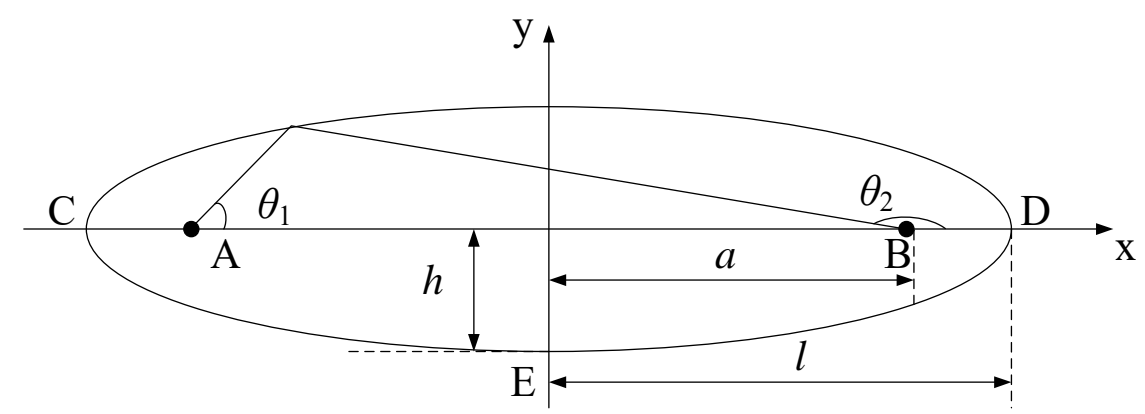

Figure 2. Overview of the Rankine body.

\subsection{Receiver Aircraft'S Fuselage and Cockpit Modelling Method}

To simplify the receiver aircraft model establishing, seem the fuselage as Rankine body [10], whose flow field is superposition by the three-dimensional point source, the three-dimensional point sink and three-dimensional straight uniform stream. Finally, the fuselage flow field model is obtained by elliptical transformation. The cockpit is modeled in the same way as the fuselage.

Assumption that the three-dimensional point source strength of Rankin body is $\mathrm{m}$ and was placed in $\mathrm{A}(-\mathrm{a}, 0)$ as shown in Fig. 2. The three-dimensional point sink strength of Rankin body is $\mathrm{m}$ and was placed in $\mathrm{B}(\mathrm{a}, 0)$. Straight uniform stream $\mathrm{U}$ is going along the $\mathrm{x}$ axis. The velocity potential and stream function can be expressed as: 


$$
\begin{gathered}
\varphi=-U x+\frac{m}{\sqrt{(x+a)^{2}+y^{2}}}-\frac{m}{\sqrt{(x-a)^{2}+y^{2}}} \\
\psi=-\frac{1}{2} U y^{2}+\frac{m(x+a)}{\sqrt{(x+a)^{2}+y^{2}}}-\frac{m(x-a)}{\sqrt{(x-a)^{2}+y^{2}}}
\end{gathered}
$$

Eq. (3) can be transformed to the form as:

$$
\psi=-\frac{1}{2} U r^{2} \sin ^{2} \theta+m\left(\cos \theta_{2}-\cos \theta_{1}\right)
$$

Cause the boundary condition rules that $\psi=0$. The function can be rewritten as

$$
\begin{gathered}
y^{2}+b^{2}\left(\cos \theta_{1}-\cos \theta_{2}\right)=0 \\
b^{2}=\frac{2 m}{U}
\end{gathered}
$$

Assuming that $\mathrm{OC}=\mathrm{OD}=1, \mathrm{OE}=\mathrm{h}$. Because of point $\mathrm{C}$ is the stationary point. The velocity of the free stream is offset by the velocity induced by source and sink.

$$
-\frac{m}{(l+a)^{2}}+\frac{m}{(l-a)^{2}}=U
$$

Cause the geometric relationship shown as in Fig. 2

$$
\left(l^{2}-a^{2}\right)^{2}=2 a b^{2} l
$$

Where $2 b^{2} \cos \alpha=h^{2}, \mathrm{Eq}(8)$ can be rewritten as

$$
\begin{gathered}
\frac{2 a}{\sqrt{h^{2}+a^{2}}}=\frac{h^{2}}{b^{2}} \\
h^{2} l \sqrt{h^{2}+a^{2}}=2 a b^{2} l
\end{gathered}
$$

Combine the two equations

$$
h^{2} l \sqrt{h^{2}+a^{2}}=\left(l^{2}-a^{2}\right)^{2}
$$

Based on the receiver aircraft's parameters. Setting the length of the fuselage is 21 , and the maximum radial width is $h$. The three-dimensional coordinates and strength $\mathrm{m}$ of point source and point sink are calculated. The induced velocity $\boldsymbol{v}_{\text {body }}$ at any point in space can be calculated as

$$
\boldsymbol{v}_{\text {body }}=\frac{m}{\boldsymbol{r}_{\mathrm{s}}}-\frac{m}{\boldsymbol{r}_{-s}}
$$

Where $\boldsymbol{r}_{s}$ is the vector from the source to the point. $\boldsymbol{r}_{-s}$ is the vector from the sink to the point. 
During refueling process, the aircraft keeps the subsonic flight. Based on the fuselage model in the incompressible flow. The Gothert Rule is used for compressibility modification. The corresponding induced velocity is obtained in the compressible flow.

While for some receiver aircraft like F/A-18, the cross section of the nose is like an ellipse. In order to obtain more accurate simulation results. The ellipse transformation is necessary.

As is shown in Fig. 3, the scale transformation is presented as

$$
x^{\prime}=x, \quad y^{\prime}=\frac{a}{b} y, \quad z^{\prime}=z
$$

Where $\mathrm{a}$ and $\mathrm{b}$ are the short axis and long axis of the ellipse. The coordinates of the desired point are converted by Eq. (13) and obtain the $v_{b o d y}{ }^{\prime}=\left[v_{x}{ }^{\prime}, v_{y}{ }^{\prime}, v_{z}{ }^{\prime}\right]^{T}$ by Eq. (12). After that, the ellipse transformation is applied again to transform $v_{\text {body }}{ }^{\prime}$ back to the original scale as

$$
v_{x}=v_{x}^{\prime}, \quad v_{y}=\frac{b}{a} v_{y}^{\prime}, \quad v_{z}=v_{z}^{\prime}
$$

Modified fuselage induced velocity shown as

$$
\boldsymbol{v}_{\text {body }}=\left[v_{x}, v_{y}, v_{z}\right]^{T}
$$

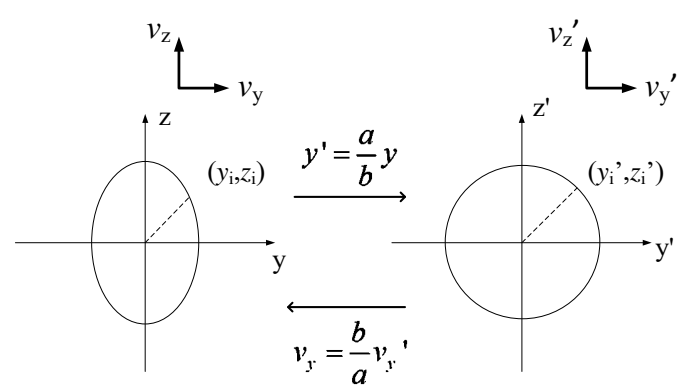

Figure 3. Illustration of the ellipse transformation

\subsection{Receiver Aircraft'S Aerofoil Modelling Method}

In the process of aerial refueling, the flight velocity is low. In this case, compared with the general CFD method, the vortex lattice method (VLM) has a small precision and high computational efficiency [11]. Under the assumption of the inviscid flow, the VLM can be used to find the flow field of the aerofoil easily and efficiently.

The VLM is a practical numerical method based on lifting surface. The VLM considers the wing as a network with a horseshoe vortex superimposed on the aerofoil. First, the aerofoil is divided into $\mathrm{M} \times \mathrm{N}$ grids, and each grid is equipped with a horseshoe vortex, and the induced velocity generated by each horseshoe vortex is obtained by the Biot-Savart Law. The ith control point's velocity induced by $\mathrm{M} \times \mathrm{N}$ horseshoe vortexes is obtained. According to the boundary conditions of control points, a set of equations of $\mathrm{M} \times \mathrm{N}$ control points can be obtained to solve the vortex strength of each horseshoe vortex, so as to obtain the induced velocity of the finite discrete horseshoe vortex on any point in space. The Gothert Rule is used for compressibility modification too. The corresponding induced velocity is obtained in the compressible flow. 


\section{The Simulation Process}

In the aerial refueling, the receiver aircraft and tanker in the same flight altitude and velocity. The receiver aircraft is close to the drogue with a stable docking velocity behind the tanker, until the connection is successful, and the relative position of the drogue is no more changes. In the process of receiver aircraft slowly close to the drogue, the bow wave effect caused by the receiver aircraft destroys the original force balance state of the drogue and produces swing. The relative position and velocity between the receiver aircraft and drogue are constantly changing. To simulate this process accurately. The digital simulation process is as follows.

(1) Calculate the equilibrium position of the hose under the no turbulence condition [12].

(2) Calculate the tension of each hose element, relative airflow velocity, external force and equivalent hose restoring force.

(3) Calculate the fuselage and cockpit's strength and coordinates of the source and sink. After that, calculate the induced velocity at the point in space.

(4) Calculate the velocity induced by the main wing on the drogue by VLM.

(5) Calculate the bow wave induced velocity at the point of the drogue by Eq. (1).

(6) The acceleration of the drogue is calculated by the induced velocity, and the acceleration of the drogue is obtained by using the fourth-order Runge-Kutta method [13].

Table 1. Parameters of the simulation.

\begin{tabular}{cc}
\hline Parameter & Value \\
\hline Tanker & $17.07 \mathrm{~m}$ \\
The length of the fuselage & $0.808 \mathrm{~m}$ \\
The maximum radius of the fuselage & $11.43 \mathrm{~m}$ \\
root chord & $4.04 \mathrm{~m}$ \\
wing sweep & $25.82^{\circ}$ \\
Drogue & \\
Weight & $200 \mathrm{~N}$ \\
Diameter & $0.6 \mathrm{~m}$ \\
\hline
\end{tabular}

\section{Simulation Results and Analysis of the Motion Characteristics of the Hose- Drogue.}

The simulation is in the condition without turbulence. The simulation parameters used in this paper are illustrated in Table 1, which are the same with the parameters in Ref. 13. Suppose that the oil filler of the probe is in $\mathrm{P}$ point. As shown in Figure 4. The body frame of the receiver aircraft $\mathrm{ObXbYbZb}$. Ob is fixed on the top of the receiver aircraft. The vertical axis $\mathrm{Xb}$ points to the tail. The vertical axis $\mathrm{Yb}$ goes straight down. The horizontal axis $\mathrm{Zb}$ is determined by the right-hand rule. The coordinates of the $\mathrm{P}$ point were shown in Table 2. In Case 1, the probe is arranged on the side of the fuselage. In Case 2-4, the probe is arranged on the top of the fuselage.

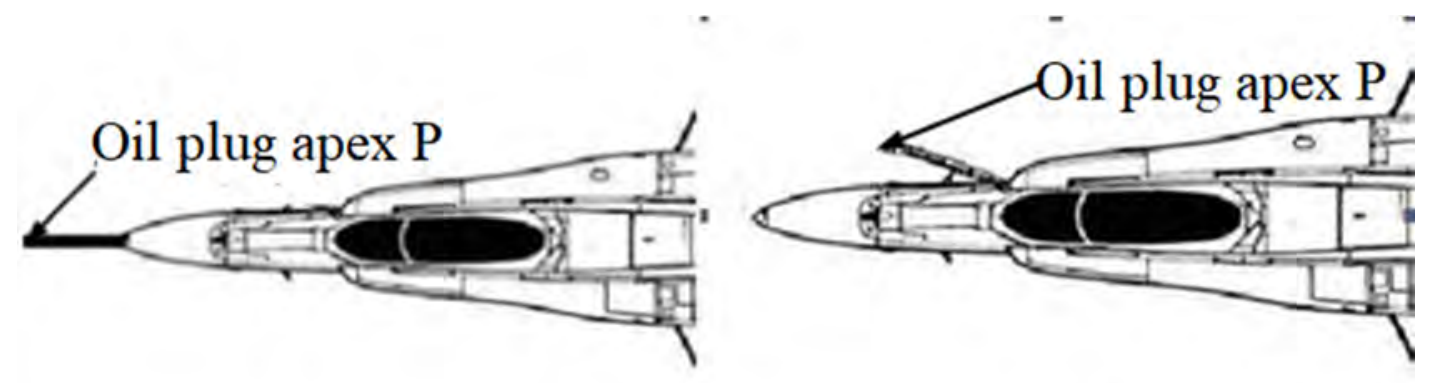

Figure 4. Illustration of the P point 
Table 2. Coordinates of the $\mathrm{P}$ point.

\begin{tabular}{cccc}
\hline Case 1 & Case 2 & Case 3 & Case 4 \\
\hline$(1.875,0.935,1.270)$ & $(1,0,0)$ & $(2,0,0)$ & $(3,0,0)$ \\
\hline
\end{tabular}

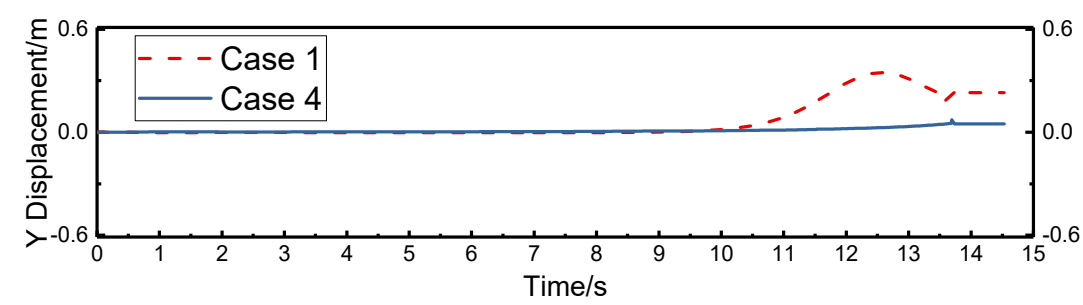

(a) Y direction

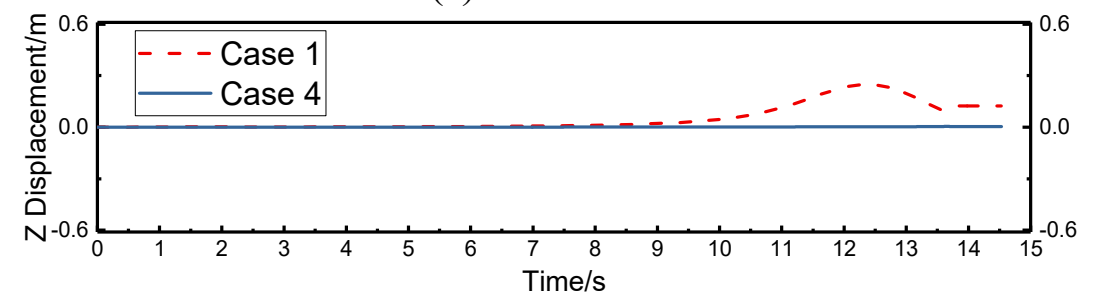

(b) $\mathrm{Z}$ direction

Figure 5. Deviation of drogue in $\mathrm{Y}$ and $\mathrm{Z}$ direction.

The deviation of the offset in the vertical and lateral of the drogue is shown in the Fig. 5. When the probe is on the side, the vertical displacement of the drogue can reach up to 0.35 meters in the entire docking process. While the lateral displacement can be as high as $0.25 \mathrm{~m}$. In contrast, when the probe is on the top, the drogue has little sway in both the lateral and vertical directions. Therefore, we can assume that when the probe is on the side, the drogue is almost not affected by the bow wave effect of the receiver during the entire air refueling docking process.

Keep the tanker's flight conditions unchanged, conduct simulation on the arrangement of the probe in the front, and take 1 meter, 2 meters and 3 meters respectively for the length of the probe. The simulation results are as follows:

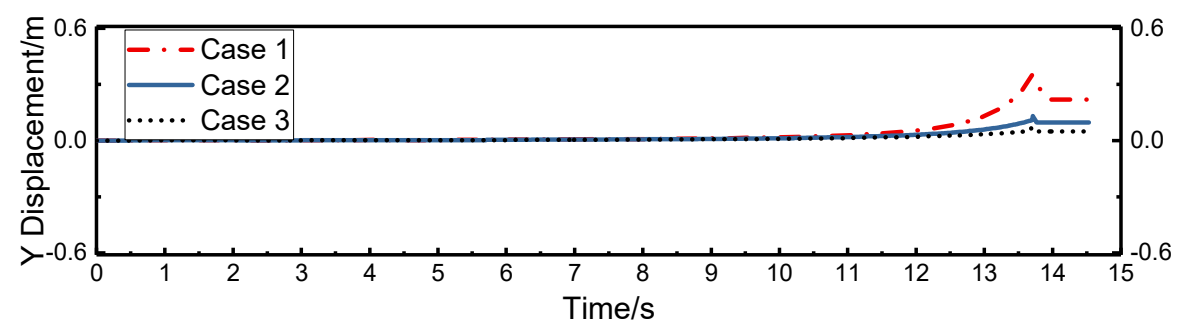

(a) Y direction

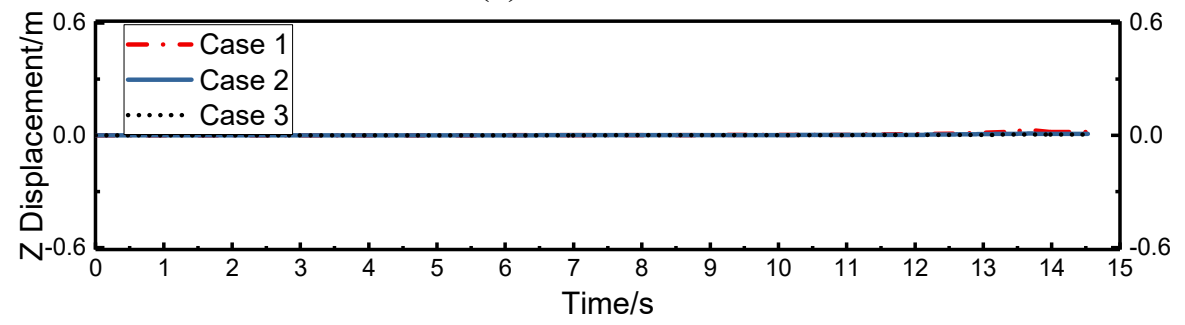

(b) $\mathrm{Z}$ direction

Figure 6. Deviation of drogue in $\mathrm{Y}$ and $\mathrm{Z}$ direction.

The deviation of the offset in the vertical and lateral of the drogue is shown in the Fig. 6. The farther from the nose is the tip P point of the probe, the smaller the vertical swing amplitude of the drogue. In the lateral direction, the amplitude of the swing is almost zero, regardless of the distance 
of $\mathrm{P}$ point from the nose. We can conclude that, the longer the probe is, the smaller the effect of bow wave on the drogue is. Although long probe can reduce the disturbance of bow wave effect, longer probe can also bring additional resistance and weight, which has a greater impact on small aircraft. Therefore, in actual engineering practice, appropriate layout mode and intubation length should be selected according to actual situation.

\section{Conclusion}

(1). In this paper, a simple and efficient modeling method is proposed based on potential flow theory, and a mathematical model of fuselage, cockpit and aerofoil is established by means of source, sink and finite discrete horseshoe vortex. Through the compressibility modification a, the model is as accurate as possible to simulate the aerodynamic characteristics of the bow wave effect.

(2). The flow field characteristics of different types of probe positions were verified. This paper analyzes the advantages and disadvantages of the two modes of arrangement of the probe on the side and the probe on the front, and summarizes their respective operating environments.

\section{Acknowledgments}

This work was financially supported by National Natural Science Foundation of China (61473307).

\section{References}

[1]. QUAN Q, WEI Z B, GAO J, et al. A survey on modeling and control problems for probe and drogue autonomous aerial refueling at docking stage[J].Acta Aeronautica et Astronautica Sinica. 2014, 35(9): 2390-2410(in Chinese).

[2]. LU Y P, YANG C X, LIU Y Y, A survey of modeling and control technologies for aerial refueling system[J].Acta Aeronautica et Astronautica Sinica. 2014, 35(9): 2375-2389(in Chinese).

[3]. Atilla Dogan, William Blake. Modeling of Bow Wave Effect in Aerial Refueling[C]. AIAA Atmospheric Flight Mechanics Conference, Toronto, Ontario Canada: AIAA, 2010.

[4]. Ujjar Bhandari, Peter R Thomasy, Steve Bullockz, Thomas S Richardson. Bow Wave Effect in Probe and Drogue Aerial Refuelling[C]. AIAA Guidance, Navigation, and Control (GNC) Conference, Boston, MA: AIAA, 2013.

[5]. Ryan P Dibley, Michael J Allen. Autonomous Airborne Refueling Demonstration Phase I FlightTest Results[C]. AIAA Atmospheric Flight Mechanics Conference and Exhibit, Hilton Head, South Carolina: AIAA, 2007.

[6]. ZHANG J. Dynamic modeling and control of the motion of the hose-drogue[D]. Nanjing: Nanjing University of Aeronautics and Astronautics, 2016:36-40(in Chinese).

[7]. WANG J, DONG X M, XU Y J, et al. Simulation and analysis of the bow wave effect of the receiver in hose-drogue aerial refueling[J]. Flight Dynamics, 2016, 34(1): 54-58(in Chinese).

[8]. Khan, J Masud. Trajectory Analysis of Basket Engagement during Aerial Refueling[C]. AIAA Atmospheric Flight Mechanics Conference, National Harbor, Maryland: AIAA, 2014.

[9]. Jennifer L Hansen, James E Murray, Norma V Campos. The NASA Dryden Flight Test Approach to an Aerial Refueling System[R]. NASA/TM-2005-212859, Edwards, California: NASA Dryden Flight Research Center, 2005.

[10]. L.M. Milne Thomson. Theoretical hydrodynamics[M]. LI Y L, YAN M W, translated. Beijing: China Machine Press, 1984.9. 545-551(in Chinese). 
[11]. QIAN Y J. Aerodynamics[M]. Beijing: Beihang University Press, 2004.9:153-165(in Chinese).

[12]. HU M Q, LIU P, NIE X, et al. Influence of air turbulence on the movement of the hosedrogue[J]. Flight Dynamics, 2010, 28(5): 20-23(in Chinese).

[13]. HU M Q, NIE X, WANG L M. Determination of hose static catenary shape in "probe-drogue" in-flight refueling system[J]. Journal of Air Force Engineering University: Natural Science Edition, 2009, 10(5): 22-26(in Chinese). 